\title{
Editorial: The Future of Nuclear Structure: Challenges and Opportunities in the Microscopic Description of Nuclei
}

\author{
Luigi Coraggio $^{1 *}$, Saori Pastore ${ }^{2 *}$ and Carlo Barbieri ${ }^{3,4,5 *}$ \\ ${ }^{1}$ Istituto Nazionale di Fisica Nucleare, Sezione di Napoli, Napoli, Italy, ${ }^{2}$ Physics Department and McDonnell Center for the Space \\ Sciences at Washington University in St. Louis, St. Louis, MO, United States, ${ }^{3}$ Department of Physics, University of Surrey, \\ Guildford, United Kingdom, ${ }^{4}$ Dipartimento di Fisica, Universita Degli Studi di Milano, Milano, Italy, ${ }^{5}$ INFN, Sezione di Milano, \\ Milano, Italy
}

Keywords: Ab initio (calculations), nuclear theory, nuclear reactions, effective field theories, many-body physics, nuclear structure

Editorial on the Research Article

The Future of Nuclear Structure: Challenges and Opportunities in the Microscopic Description of Nuclei

\section{OPEN ACCESS}

Edited and reviewed by: Sonia Bacca,

Johannes Gutenberg University Mainz, Germany

*Correspondence: Luigi Coraggio

coraggio@na.infn.it

Saori Pastore

saori.pastore@gmail.com

Carlo Barbieri

carlo.barbierio@unimi.it

Specialty section:

This article was submitted to

Nuclear Physics,

a section of the journal

Frontiers in Physics

Received: 07 November 2020

Accepted: 20 November 2020

Published: 05 February 2021

Citation:

Coraggio L, Pastore $S$ and Barbieri C (2021) Editorial: The Future of Nuclear

Structure: Challenges and

Opportunities in the Microscopic

Description of Nuclei.

Front. Phys. 8:626976.

doi: $10.3389 /$ fphy.2020.626976
The past two decades have witnessed tremendous progress in the microscopic description of atomic nuclei. Within this approach, nuclei are described in terms of nucleons interacting via realistic twoand three-body forces, constrained to accurately reproduce a large body of data for few nucleons systems. The goal of the nuclear theory community is to gain an accurate and predictable understanding of how the properties of many-body systems, along with their dynamics and structure, emerge from internucleon correlations induced by the strong interaction.

Progress in the microscopic (or, ab initio) theory has been quite notable and it has been supported by two major pillars: First, thanks to the advent of Effective Field Theories (EFTs), we can now systematically develop nuclear Hamiltonians that are rooted in the fundamental properties and symmetries of the underlying theory of QCD. Second, advances in computational resources and novel powerful algorithms allow us to solve 1) the many-nucleon problem efficiently, and 2) quantify the degree of reliability of theoretical calculations and predictions. In many cases, microscopic computations achieve an accuracy that is comparable or superior to the precision delivered by current EFT interactions. This sparked a renewed interest to further broaden the focus of ab initio theory and address open problems in nuclear physics.

While the status of the first pillar has been recently discussed by "The Long-Lasting Quest for Nuclear Interactions: The Past, the Present and the Future" Topical Review on this Journal, here we focus on the exciting new developments in microscopic theory. At present, ab initio computations of nuclear structure include up to medium-mass isotopes. The heaviest systems currently reached-with different degrees of accuracy-have mass number $A \approx 140$. These computational limits are constantly being pushed forward. At the same time, the community is expanding into new directions, in particular toward the study of electroweak observables and nuclear reactions, that nowadays require predictions with an accuracy never reached before for similar mass ranges.

In collecting the contributions for this Research Topic, we sought to gather contributions from authors who could summarize the current state-of-the-art microscopic calculations in Nuclear Theory, favoring a selected but broad view over an attempt to cover every application. All presented contributions stem from well-established methods in computational nuclear structure, and indicate recent theoretical advances and prospective outlooks, challenges and opportunities for Nuclear 
Theory. Most importantly, it is our hope that this collection will confer a big picture, including references to basic material, that will be valuable for young researches who intend to enter this exciting discipline.

The richness of applications in modern $a b$ initio nuclear theory can be appreciated in Hergert's contribution that provides us with a general overview of the most successful microscopic many-body approaches currently in use. Traditionally, the refinement and sophistication of these computational tools has given fundamental support to advance the theories of nuclear forces. Quantum Monte Carlo (QMC) techniques allow to solve the many-body Schrödinger equation with high accuracy for light nuclei up to masses A 16-40. Gandolfi et al. discuss the use of QMC methods (namely, Variational, Green's Function, and Auxiliary Diffusion Monte Carlo methods) in combination with local chiral interactions in coordinate space. QMC methods are used in lattice effective field theory, where the EFT Lagrangian is implemented in momentum space with nucleons and pions placed on a lattice. Lee discusses the basic features of this approach and its high potential for understanding clustering phenomena.

For heavier isotopes, $a b$ initio theories can be pushed to masses A $\sim 140$ provided that one retains only the relevant nuclear excitations, as it is done through all-orders resummations. Among these methods, the self-consistent Green's function (SCGF) theory gives direct access to the spectral information probed by a wide range of experiments as reviewed in detail by Somà's contributions. Once in the region of the nuclear chart that corresponds to medium masses, open shell isotopes become the next challenge to be addressed by the theory. In fact, resolving the degeneracy in uncorrelated systems requires large scale configuration mixing. Coraggio and Itaco demonstrate how this can be handled by projecting the correlated many-body states into a shell model Hamiltonian, using the so-called "Q-box" formalism. A similar strategy is shared by other computational frameworks, such as coupled cluster and in-medium SRG, that are touched upon in the contribution by Hergert. A less conventional approach to open shells is to break SU(1) symmetry (in short, allowing for breaking particle number conservation). This is discussed by Somà within SCGFs and by Tichai et al. in the framework of many-body perturbation theory.

The remainder of this topical review focuses on selected open challenges in Nuclear Theory that require an $a b$ initio approach.
Two contributions show different aspect of studying infinite nucleon systems and the implications for astrophysical scenarios. Tews covers QMC calculations of the equation of state $(\mathrm{EoS})$ of dense matter in neutron stars. With the recent observation of star mergers and the birth of multi-messenger astronomy, it has become of prime importance to understand the finite temperature properties of the EoS. Rios discusses this topic and how the structure of neutron matter depends on temperature, using SCGF theory.

In the quest for physics beyond the Standard Model, Nuclear Theory, and in particular accurate calculations of neutrinonucleus interactions at all energy scaler, plays a crucial role. This is carefully analyzed by Rocco's contribution that address this challenge with emphasis on impacts to neutrino oscillations experimental programs. The last contribution of this Topical Review addresses one of the hardest open challenges in the interpretation of experimental data: the lack of a truly firstprinciples theory that can describe consistently both structure and reaction processes. Rotureau highlights recent steps in deriving an $a b$ inito optical potential using the coupled cluster method (that, together with SCGF, is one of the two possible approaches to this problem).

We are really grateful to all the scientists participating in this project and hope that the reader will enjoy this Topical Review.

\section{AUTHOR CONTRIBUTIONS}

All authors listed have made a substantial, direct, and intellectual contribution to the work and approved it for publication.

Conflict of Interest: The authors declare that the research was conducted in the absence of any commercial or financial relationships that could be construed as a potential conflict of interest.

The handling editor declared a past co-authorship with one of the authors SP.

Copyright (c) 2021 Coraggio, Pastore and Barbieri. This is an open-access article distributed under the terms of the Creative Commons Attribution License (CC BY). The use, distribution or reproduction in other forums is permitted, provided the original author(s) and the copyright owner(s) are credited and that the original publication in this journal is cited, in accordance with accepted academic practice. No use, distribution or reproduction is permitted which does not comply with these terms. 